\title{
“One-Button” Wear Debris Analysis
}

\author{
R. J. Lee*, H. P. Lentz*, D. G. Kritikos*, and A. M. Toms** \\ * R. J. Lee Group, 350 Hochberg Road, Monroeville, PA 15146 \\ ** JOAP-TSC, 85 Millington Avenue, Pensacola, FL 32508-5010
}

Modern Computer-Controlled Scanning Electron Microscopy (CCSEM) permits most aspects of a particle analysis to be performed in an automated, unattended manner. However, in spite of the high degree of automation, there is still a component of operator set-up required prior to starting the analysis: the location and shape of the sample(s) must be set up, focus and brightness/contrast must be set, and "run parameters" must be chosen. These run parameters describe the microscope settings, elements of interest, time and size criteria, and other analysis control settings. It requires an individual of at least moderate SEM expertise to perform this setup correctly and repeatably.

In order to make the target system of this study (a Personal SEM or "PSEM," produced by Aspex LLC) perform as a dedicated Wear-Debris analyzer, several design goals had to be achieved:

1) The system needs to be capable of being run by non-traditional operators. Such individuals will not have had any classical SEM training, and will be using the system on a transient basis, which does not make it cost- or time-effective to provide such training.

2) A summary report needs to be generated for each sample group, while all images, spectra, and numerical information need to be retained in a searchable data repository.

3) The entire setup must be packaged for reliable field-deployability.

The ideal would be to remove all microscope set-up steps from the responsibility of the operator, and permit a simpler, "one-button" start-up. In this way, the operator would simply load his or her samples, enter sample ID information in the database, and tell the system to "go".

In order to achieve this, the operator-intensive steps needed to be established and stored before routine analysis could be performed. To this end, a sample tray was defined based on the size and shape of the sample. The samples can only be placed in pre-defined areas. By knowing where samples will be located, and the size and shape of the samples, the stage-setup can be saved to disk once during calibration, along with optimal conditions of beam, working distance, brightness and contrast, and focus. These calibrations can then be recalled by the computer automatically for each analysis run. As an adjunct to this, automated procedures were designed to fine-tune the beam, focus, and brightness/contrast on a specialized standard sample built into the stage mechanism. This model also supports multiple calibrations for different sample types.

The PSEM was then configured with an external computer as part of a client-server topology (Figure 1). Resident on this external computer is a database, user interface, and PSEM-interface module. The user interacts with this less "threatening" computer to enter sample identification information in a menu-type interface. He or she then clicks a button to initiate the analysis. The database computer passes the setup information to the PSEM, and instructs it to begin the analysis. The PSEM recalls all the pre-programmed calibrations in lieu of ordinary interactive microscope setup by the operator, and at that point will invoke the automated procedures. 
Thus the non-traditional operator need not interact with the microscope controls to start a run. As the analysis proceeds, all data is shipped to the database computer, where it is processed and stored. When complete, the database creates a summary report, which will indicate pass/fail conditions based on pre-programmed criteria, along with a summary of particle types found.

For most purposes, this is the end of the analysis, and the operator can start the process anew. If, however, there is an anomaly that warrants further investigation, the database can be queried to display individual particle images and spectra as well as data tables. Since the data and images are in electronic form, they can be shared to remote locations with scientists and engineers to whom particle shape and composition may provide insights that are not readily discernible in the summary report. Thus we may preserve the essence of the SEM - its imaging capability - while providing a streamlined process for routine analysis and simplified distillation of results.

The SEM and peripherals described above have been built into a rugged enclosure for deployment. The system is shock-mounted and configured such that the stage, gun, and other components are accessible via hinged doors. The SEM monitors are replaced with flat-screens; since these monitors are mainly for maintenance they do not need to be as large and clear as those on a conventionally-operated SEM. Everything in the enclosure is powered by a single electrical cord which plugs into a standard 110 volt outlet.

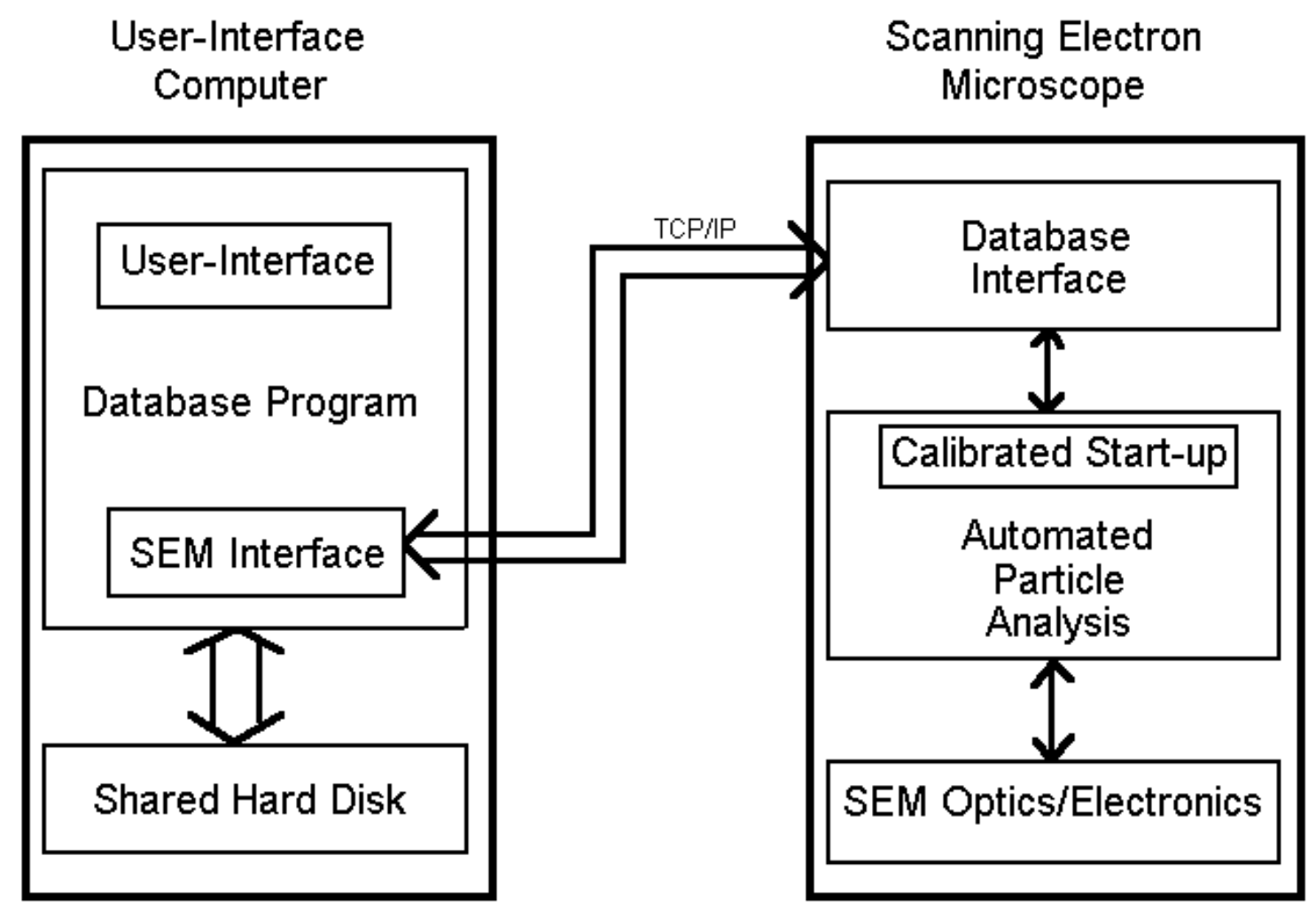

Figure 1 -- System Topology 